\title{
Metallic vaginal ring pessary: A rare entity
}

\section{Uprety DK ${ }^{1}$, Regmi MC ${ }^{2}$, Budhathoki $\mathbf{B}^{2}$, Tiwari RR ${ }^{2}$, Subedi $\mathrm{S}^{2}$, Chhetri $\mathrm{S}^{2}$}

${ }^{1}$ Additional Professor, ${ }^{2}$ Senior resident, Department of Obstetrics and Gynaecology, B.P. Koirala Institute of Health Sciences, Dharan, Nepal

\begin{abstract}
Vaginal pessaries still have role in the management of uterovaginal prolapse especially in elderly women, unfit for surgery and as a temporary relief for women waiting for surgery. Forgotten vaginal pessaries may cause serious complications. We here by present a case wtih metallic vaginal ring pessary for 16 years without significant symtoms.
\end{abstract}

Key words: Prolapse, Ring pessary, Metallic pessary

$\mathrm{U}$ terovaginal prolapse is very common morbidity among Nepalese women. The incidence of genital prolapse is difficult to determine, as many women do not seek medical advice. It has been estimated that a half of parous women lose pelvic floor support, resulting in some degree of prolapse, and among these women $10-20 \%$ seek medical care ${ }^{1}$. Patient may present with mass protruding per vaginum, abnormal discharge or bleeding per vaginum, urinary or bowel symptoms and low backache. Though it is widely prevalent in women residing both in hills and flat lands, the prolapse surgery facility is available only in some regional hospitals and the big cities in Nepal. Only very recently the surgical treatment has become more available due to mobile prolapse surgery camps and opening of medical colleges in the periphery. Majority of women with this condition either live with suffering or use rubber ring pessaries as a temporary relief. It is very important to explain to the patient that the pessaries need to be changed every three months and earlier if she gets pain, excessive or foul smelling discharge or bleeding per vaginum or urinary symptoms. Usually it is well tolerated and does not cause serious morbidities, but if instructions are not followed or forgotten, it may lead to serious complications like ulceration, fistula formation, urinary symptoms or urosepsis. We are reporting a case of metallic ring pessary used for 16 years with out significant symptoms.

\section{Case report}

A 42 year-old lady presented to gynaecological out patient department of BP Koirala Institute of Health Sciences with mass coming out per vaginum for last 20 years. This was small initially, had grown slowly and remained out of introitus for about last 16 years and was causing difficulty in household work and low back ache. At times she used to get difficulty in voiding which required the repositioning of the bulge but never suffered from urinary retention. History of intermittent vaginal discharge was present but it was not blood stained or foul smelling. Her menstrual cycles were regular. She was $\mathrm{P}_{4} \mathrm{~A}_{0} \mathrm{~L}_{4}$, last child being 22 year old; all were full term normal home deliveries. She underwent tubal sterilization 22- year back.

About 16 years back she had visited Eastern Regional Hospital at Dharan for the same problem and a ring pessary was inserted. With this her symptoms disappeared. After about six months the ring pessary came out in pieces. She was not advised to change the pessary every three months or to come for follow up. Again she developed protrusion of mass per vaginum causing difficulty in walking and voiding. After about two months of these symptoms (about 15 years back) she visited the same nurse, who treated her previously. Again the ring pessary was inserted and she become alright. She does not know about the nature of the pessary inserted. But six months later she noticed that the mass again started coming out of vagina. She didn't notice ring falling out. No history of excess discharge per vaginum, no history of burning micturation, increase in urinary frequency, urinary leakage or difficulty in bowel movement. 
On examination she was not pale; her vital signs were within normal limit. Abdominal examination revealed normal findings, on local examination: grade III cystocele with small area of keratinization, with third degree uterine descent, enterocele and rectocele. On palpation a ring like structure was felt around the cervix, which was totally covered by vaginal epithelium and no ulceration or scar was visible (Figure 1). Uterus was retroverted and normal in size.

She was advised for exploration for foreign body and prolapse surgery.

Vaginal hysterectomy with anterior colporrhaphy and posterior colpoperineorrhaphy was done. Transverse incision above the cervix revealed a metallic foreign body (Figure 2). Incision was extended laterally on both sides, this revealed an aluminium bangle which was cut with pliers at one place and removed by sliding the bangle (Figure 3 ). Rest of the procedure was carried out as standard Ward Mayo procedure. Her post operative recovery was uneventful except that she had voiding difficulty and needed re catheterization on third day. Catheter was removed on seventh post operative day; she had no voiding difficulty and was discharged from the hospital. She was doing well three months after the surgery.

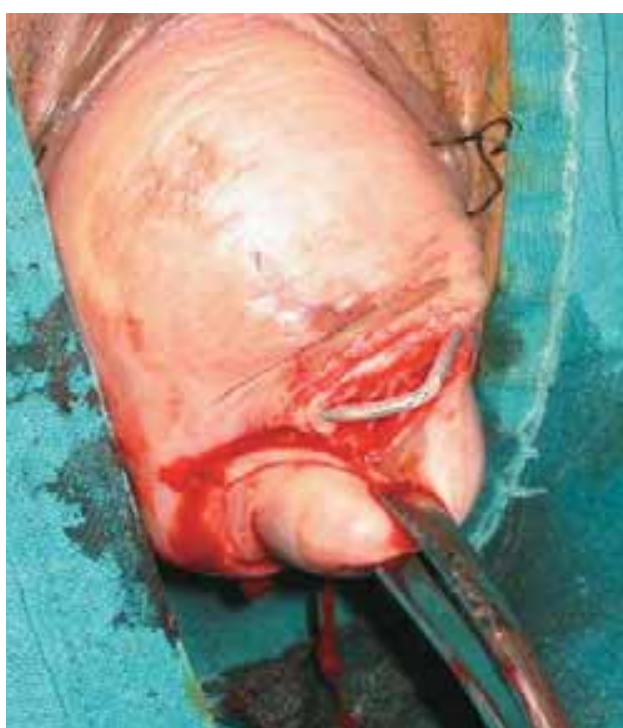

Fig 2: Metallic ring exposed at incision site

Fig 1: Circular band seen around cervix.

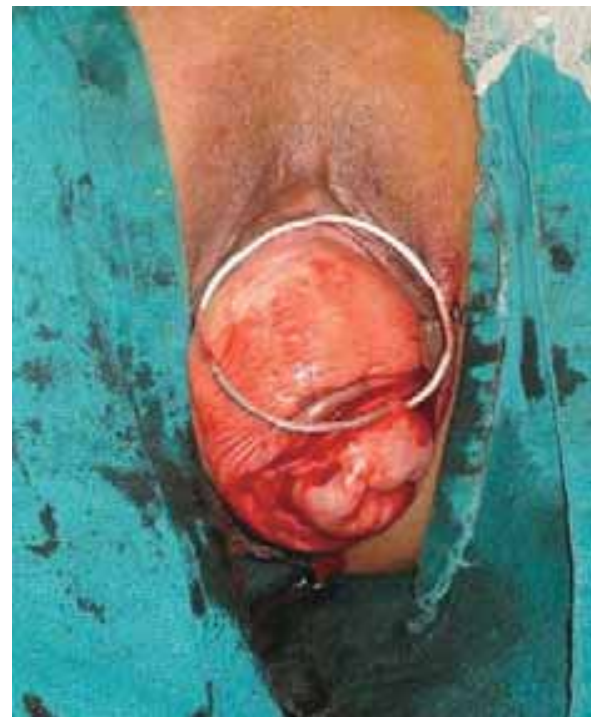

Fig 3: Metallic ring after removal 


\section{Discussion}

In general pessary treatment of genital prolapse is technically easy and quick procedure. Most of the time use of pessary is not associated with serious complications. In Nepal mostly rubber ring pessaries are used for this purpose. In our setup, it is not uncommon to find small rubber balls, ragged clothes, clay balls in the vagina, inserted by patient them selves or by traditional healers to reduce the prolapse. Our case is unusual in two aspects: use of metallic ring as pessary (ordinary bangle) and in spite of being there for long duration, it was not causing much discomfort or any serious problem. Though the metallic ring must have caused ulceration at some time in the past and later got completely embedded inside the vaginal mucosa, it had not eroded into the urinary bladder or rectum. When it gives symptomatic relief and does not cause any discomfort, women tend to forget it. Forgotten ring pessaries can erode into the rectum causing rectovaginal fistula ${ }^{2,3}$; erode into the urinary bladder causing vesicovaginal fistula ${ }^{4,5}$, urosepsis $^{6}$, urinary symptoms like frequency and urge incontinence ${ }^{7}$.

\section{Conclusion}

It is very essential to explain the patient about what is going to be done and to show what is being inserted and how often to change or to come for follow up. Caring physician should do proper per speculum examination under good light when patient presents with foul smelling discharge, abnormal bleeding, urinary or bowel symptoms to rule out the possibilities of forgotten pessaries and its sequels.

\section{References}

1. Thakar R, Stanton S. Management of genital prolapse. BMJ 2002;324:1258-62.

2. Hanavadi S, Durham-Hall A, Oke T, Aston N. Forgotten vaginal pessary eroding into rectum. Ann R Coll Surg Engl 2004;86:1-2.

3. Kankam OK, Geraghty R. An erosive pessary. J R Soc Med 2002;95:507.

4. Grody M H T, Nyirjesy P, Chatwani A. Intravesical foreign body and vesicovaginal fistula: A rare complication of neglected pessary. Int Urogynecol J 1999;10:407-8.

5. Wheeler LD, Lazarus R, Torkington J, O'Mahony M S. Perils of pessaries. Age and ageing 2004;33:510-1.

6. Roberge RJ, McCandiljsh MM, Dorfsman ML. Urosepsis associated with vaginal pessary use. Ann Emerg Med 1999;33:581-83.

7. Chou Y C, Yu K J. Entrapped vaginal pessary presented with frequency and urge incontinence. J Chin Med Assoc 2003;66:181-3. 